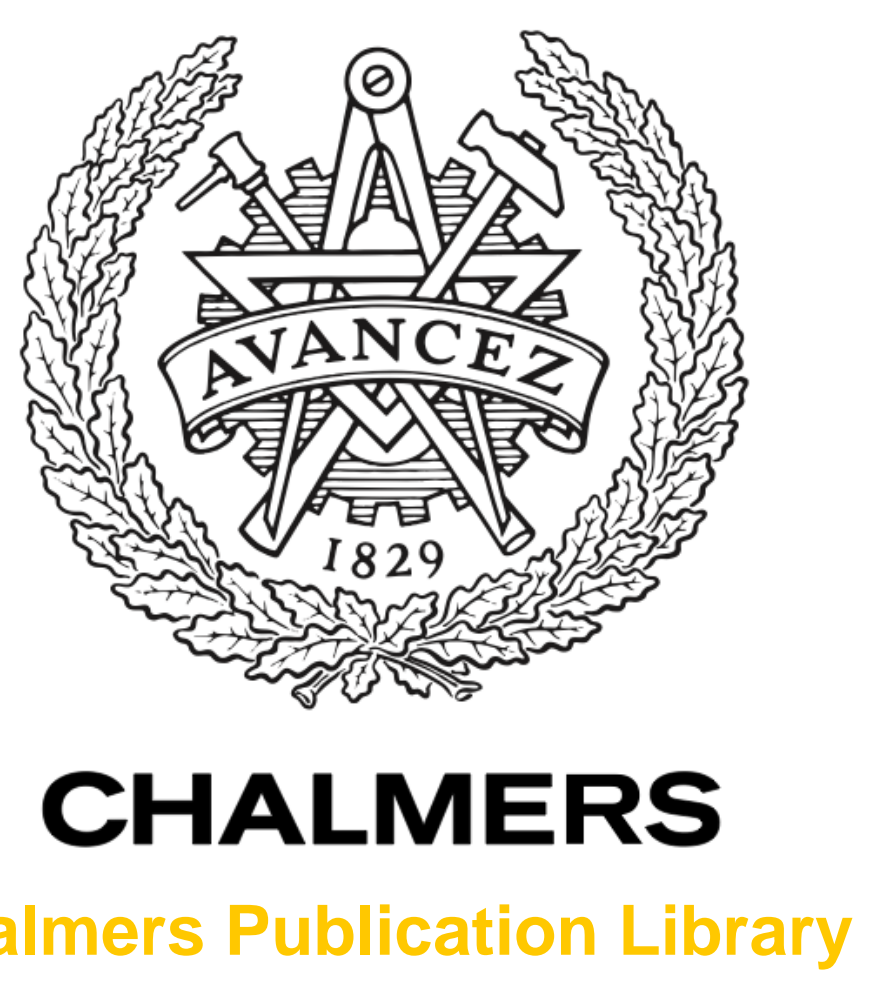

Chalmers Publication Library

\title{
Design of integrated diplexer-power divider
}

This document has been downloaded from Chalmers Publication Library (CPL). It is the author's version of a work that was accepted for publication in:

IEEE MTT-S International Microwave Symposium Digest, IMS 2016; San Francisco; United States; 22-27 May 2016 (ISSN: 0149-645X)

Citation for the published paper:

Sorkherizi, M. ; Vosoogh, A. ; Kishk, A. et al. (2016) "Design of integrated diplexer-power divider". IEEE MTT-S International Microwave Symposium Digest, IMS 2016; San

Francisco; United States; 22-27 May 2016, vol. 2016-August

http://dx.doi.org/10.1109/MWSYM.2016.7540124

Downloaded from: http://publications.lib.chalmers.se/publication/246226

Notice: Changes introduced as a result of publishing processes such as copy-editing and formatting may not be reflected in this document. For a definitive version of this work, please refer to the published source. Please note that access to the published version might require a subscription.

Chalmers Publication Library (CPL) offers the possibility of retrieving research publications produced at Chalmers University of Technology. It covers all types of publications: articles, dissertations, licentiate theses, masters theses, conference papers, reports etc. Since 2006 it is the official tool for Chalmers official publication statistics. To ensure that Chalmers research results are disseminated as widely as possible, an Open Access Policy has been adopted.

The CPL service is administrated and maintained by Chalmers Library. 


\title{
Design of Integrated Diplexer-Power Divider
}

\author{
Milad Sharifi Sorkherizi ${ }^{1}$, Abbas Vosoogh ${ }^{2}$, Ahmed A. Kishk ${ }^{1}$, and Per-Simon Kildal ${ }^{2}$ \\ ${ }^{1}$ Electrical and Computer Engineering Department, Concordia University, Montreal, Quebec, Canada \\ ${ }^{2}$ Department of Signals and Systems, Chalmers University of Technology, Gothenburg, Sweden
}

\begin{abstract}
A new configuration is introduced to integrate diplexers and power dividers. The proposed configuration is based on coupling matrix. The design of the lumped element network is based on addition of an extra term to the conventional error function of the coupling matrix to decouple the two ports of the power divider. An optimized lumped element network is implemented successfully on an EBG based guiding technology known as ridge gap waveguide. The optimization of the physical structure is done efficiently by dividing the diplexer-power divider into many sub-circuits and analyzing the corrected delay response of them.

Index Terms - Diplexer, power divider, coupling matrix, antenna array.
\end{abstract}

\section{INTRODUCTION}

Diplexers and power dividers are two important and integral part of many communication systems. Several geometries and design procedures are introduced to improve their functionality in terms of size reduction, insertion loss, and rejection. As the design and implementation of the diplexers are more involved, considerable resources could be found in the literature [1]-[3]. One novel geometry that contributes to the performance and size reduction of the system is integrating the power divider inside the diplexer to be common with the antenna array's corporate-feed. In this paper, we propose all-pole coupledresonator diplexer-power divider. We introduce a systematic design procedure. In order to achieve this, a novel optimization technique is used. The optimized lumped element network is implemented on an electromagnetic band gap (EBG) based guiding technology known as ridge gap waveguide [4].

In the section II of the paper, a new error function is introduced to design the coupling matrix $(\mathrm{CM})$ of the diplexerpower divider network. Also, a general recipe is explained to ensure the convergence of the optimization procedure. The proposed error function removes the coupling between the two power-divider output ports and simultaneously design the channel filters correctly based on an equi-ripple performance. The method is tested on a seventh order channel diplexer successfully. In the final section of the paper, the optimized CM of the seventh order channel diplexer-power divider is implemented on a real guiding structure. Converting the lumped element model of a diplexer to the physical dimensions is generally considered a difficult task, since it is mainly based on optimization procedures. In [5] a method is introduced to design bandpass filters using the corrected delay response of the sub-circuits. We used this method with some modifications in order to convert the CM model of diplexer-power divider to the physical dimensions of the structure without using any fullwave optimization. This is done successfully using ridge gap and groove gap waveguide technology. In brief, gap waveguide technology can be explained as a new wave-guiding mechanism that uses a periodic band gap geometry around a guiding ridge to control the power flow direction. As such, a high Q-factor quasi TEM waveguide is achieved without a need for any electrical contact between the separate parts of structure. The simulated response of the diplexer-power divider agrees very well with CM model. The proposed device is integrated easily with the corporate feed of the antenna array. The proposed technique allows for the design of a high performance module that integrate the diplexer with the radiating elements efficiently with almost similar size to the antenna. The complete design of the diplexer and antenna will be presented in the future publications.

\section{GEOMETRY AND DESIGN OF DIPLEXER-POWER DIVIDER}

The coupling diagram of an integrated $7^{\text {th }}$ order channel diplexer-power divider is shown in Fig. 1. Every channel filter is composed of seven resonators intercoupled to each other. Two channels are combined using a distributing node (Resonator 0 ). The distributing node is coupled to two outputs which represents the power divider. It is required for the power to be divided equally in the outputs of the circuit. Thus, output couplings are equal in port 3 and 4 that is shown with $R_{o}$. In order to calculate the coupling values and resonant frequency of the nodes, one can use the optimization routine outlined in [6]. In that case, an error function should be constructed to enforce the position of poles and zeros of the system along with the passband edges and the ripple of Chebyshev function. This procedure yields correct $\mathrm{CM}$ in case of traditional diplexers as shown in [7]. Here, the integration of the diplexer with the power divider creates a spurious coupling between Port 3 and Port 4 through Resonator 0 . This unwanted resonance has severe adverse effect on the isolation of channels and has be removed from the response of the system. To do so, the pole in $\mathrm{S}_{33}$ should be removed. This can be done by enforcing a transmission zero in $\mathrm{S}_{43}$ at the center frequency of the diplexer which can be achieved by adding an extra term to the conventional error function as follow: 


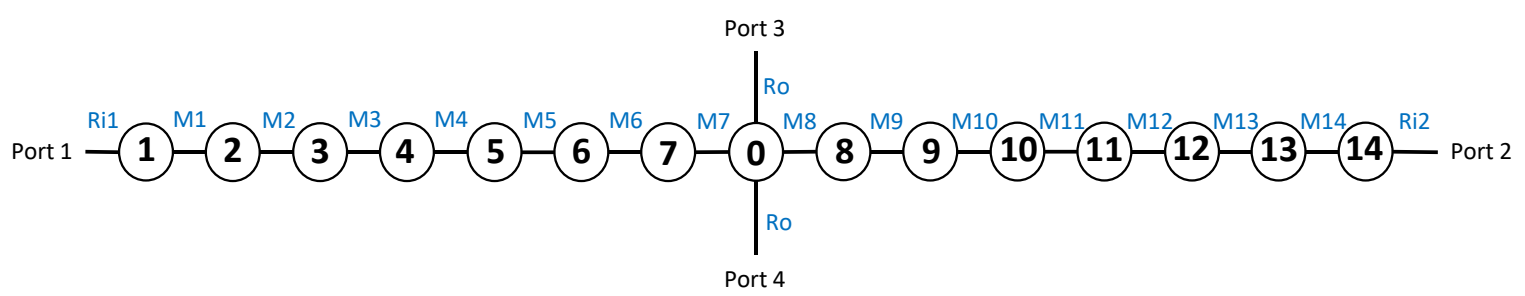

Fig. 1. Coupling diagram of a $7^{\text {th }}$ order channel integrated diplexer-power divider.

$$
\begin{aligned}
\operatorname{errf}=\sum_{1}^{n 1} \mid S_{11}( & \left.f_{p}^{i}\right)\left.\right|^{2}+\sum_{1}^{2}\left(\left|S_{11}\left(f_{e}^{i}\right)\right|-\varepsilon\right)^{2} \\
& +\sum_{n 1+1}^{n 2}\left|S_{22}\left(f_{p}^{i}\right)\right|^{2} \\
& +\sum_{3}^{4}\left(\left|S_{22}\left(f_{e}^{i}\right)\right|-\varepsilon\right)^{2}+\left|S_{43}\left(f_{c}\right)\right|^{2}
\end{aligned}
$$

where $f_{p}$ are the poles positions, $f_{e}$ are the start and end frequencies of the passbands, and $\varepsilon$ is the ripple factor. The center frequency of the diplexer $\left(f_{c}\right)$ is defined by:

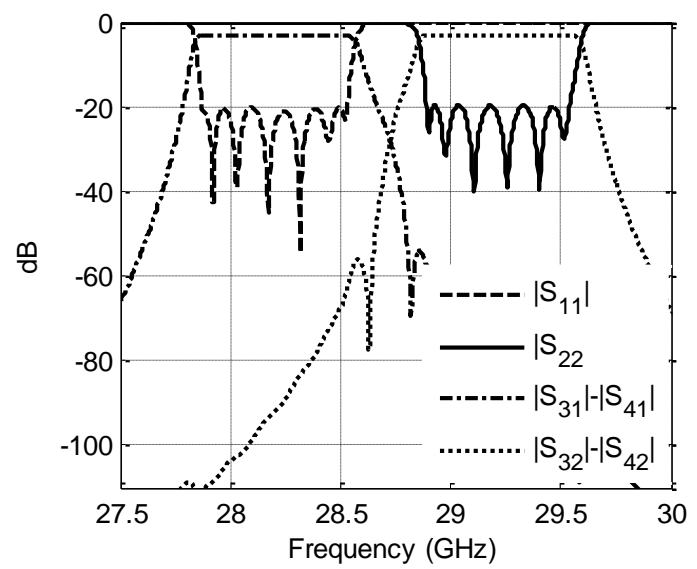

(a)

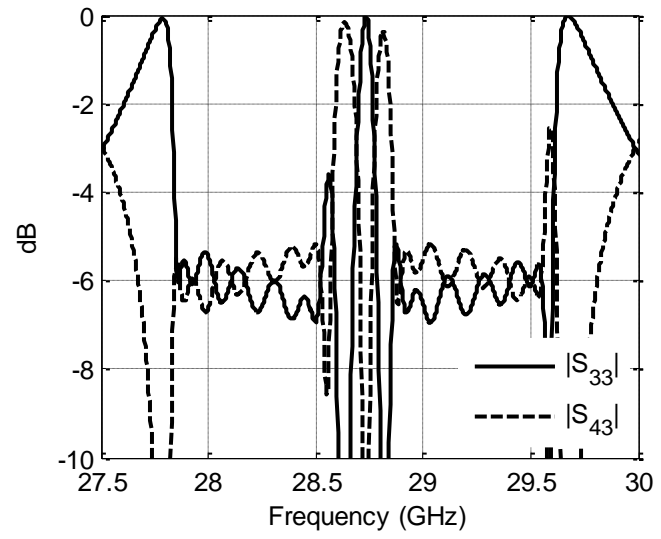

(b)

Fig. 2. Response of the optimized CM of the integrated diplexerpower divider.

$$
f_{\mathrm{c}}=\sqrt{f_{e}^{1} f_{e}^{4}}
$$

As the configuration in Fig. 1 normally is unable to create any transmission zero in finite frequencies, therefore, the term regarding them is absent in the error function. The poles $f_{p}{ }^{l}$ to $f_{p}^{n l}$ represents the poles of filter in Channel 1 and the $f_{p}{ }^{n I+1}$ to $f_{p}^{n 2}$ are the poles of Channel 2 filter. Also $f_{e}{ }^{l}$ and $f_{e}{ }^{2}$ are the passband edge frequencies of Channel 1. In the same way, $f_{e}^{3}$ and $f_{e}^{4}$ represent the passband of Channel 2. It's important to note that even though the CM model is solved very fast, since the number of the unknowns is extremely big, it is important to start the optimization with the correct initial values. To do so, coupling matrix of Chebyshev filter for each channel is used as the initial point. For the output coupling of the diplexer (Port 3 and 4 to Resonator 0 ), normalized coupling of $M=1$ is used as the initial value. Also, Resonator 0 is set to the center frequency of the diplexer $\left(f_{c}\right)$.

Here, it is intended to have channels with center frequencies at $28.2068 \mathrm{GHz}$ and $29.2148 \mathrm{GHz}$ with $650 \mathrm{MHz}$ bandwidths with $20 \mathrm{~dB}$ return loss. Using the error function in (1) and considering the outlined guidelines, the coupling values and resonant frequencies of the diagram of Fig. 1 is calculated. The response of the CM is plotted in Fig. 2(a). Using the improved error function in (1), no spurious pole is present in the middle of the band. The plotted magnitude of $S_{33}$ and $S_{43}$ also confirm this fact by showing a zero at the exact same frequency (Fig. 2(b)). If the optimization of the CM was done using the conventional methods, a very strong resonance appears at $f_{c}$ that has adverse effect on the isolation between the channels.

\section{REALIZATION OF THE INTEGRATED DIPLEXER-POWER DIVIDER IN RIDGE GAP WAVEGUIDE}

The optimized CM of the diagram in Fig. 1 is to be realized using gap waveguide technology. The geometry is shown in Fig. 3. The gap waveguide consists of a semi-periodic texture that along with the upper plate creates a forbidden electromagnetic band. The EBG texture surrounds the guiding ridges and the cavities. Therefore, the energy confides over the air gap between the ridge and the top plate and inside the cavities. As the result, gap waveguide technology doesn't need any electrical contact between the lower part and the top plate. Thus, it's a suitable guiding medium for high frequency bands 


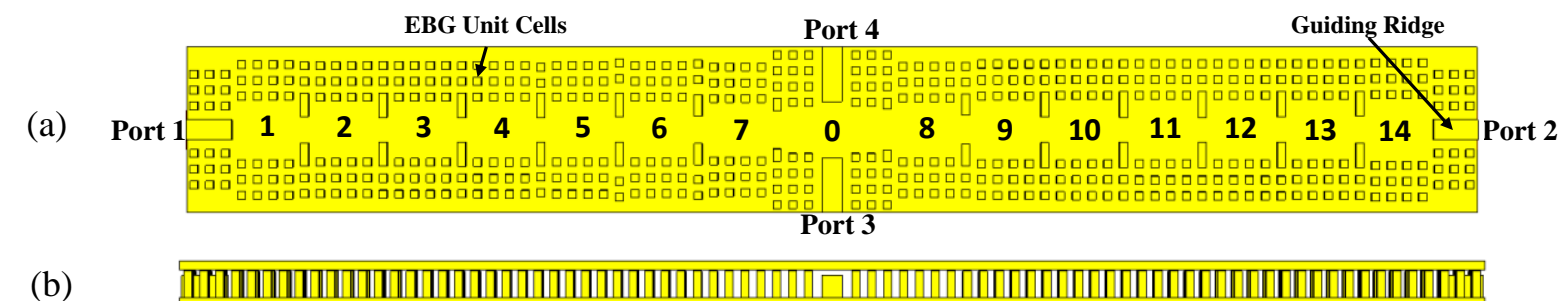

Fig. 3. Integrated diplexer-power divider using gap waveguide technology. a) top view without the upper lid, b) side view.

where electrical contact is difficult and expensive to realize due to small size of the components.

Gap waveguide generally is known as a computationally expensive structure, therefore the realization of the $\mathrm{CM}$ on the structure in Fig. 3 should be done by an efficient and fast procedure without using any full-wave optimization. Here, we used the delay response of the sub-circuits and space mapping to convert the diagram of Fig. 1 into cavity and iris dimensions of Fig. 3. This method was proposed in [5] for bandpass filters, but with some modification it is adoptable for diplexers as well. The structure is divided into three sub-circuits, which are excited at Port 1, 2 and 3 separately. Group delay response of $S_{11}, S_{22}$ and $S_{43}$ is used to extract the CM parameters of each sub-circuit individually. Later, using a linear mapping, the correct dimensions are calculated in an iterative procedure. CST Microwave Studio is used for all EM simulations. The optimized response of the design is shown in Fig. 4, which shows an excellent agreement with the $\mathrm{CM}$ model. Using Aluminum as the constructing material, a minimum of $0.9 \mathrm{~dB}$ insertion loss is observed in each of the outputs at both channels.

The designed diplexer-power divider will be integrated with the corporate-feed of a $16 \times 16$ antenna array that is constructed in the same technology. The circuit is placed in the middle of the array and feeds each half of the array equally using the

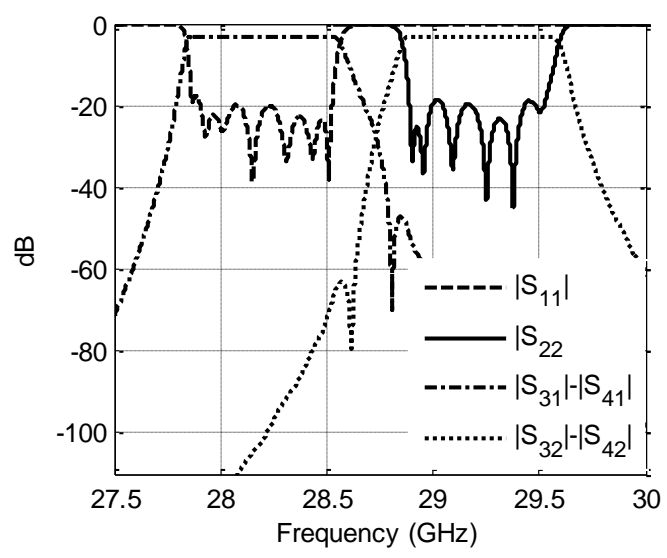

Fig. 4. Simulated EM response of the integrated diplexer-power divider. output of the divider. Therefore a very compact module is achieved.

\section{CONCLUSION}

A design procedure for a novel geometry has been presented to realize an integrated diplexer-power divider. The lumped model of the design is based on coupling matrix and is achieved using a new error function which is capable of removing the coupling between the two outputs of the circuit. A guideline has been proposed to design the CM efficiently. Realization of the $\mathrm{CM}$ on an EBG based guiding medium has been carried out using a novel method, based on dividing the structure into many sub-circuits and using the delay responses of them in combination with space mapping. Final optimized EM response has been computed, which has shown an excellent agreement with the CM response. The presented geometry has a specific application in antenna array modules.

\section{REFERENCES}

[1] J. Rhodes and R. Levy, "A Generalized Multiplexer Theory," IEEE Trans. Microw. Theory Tech., vol. 27, no. 2, pp. 99-111, Feb. 1979.

[2] A. Morini, T. Rozzi, and M. Morelli, "New formulae for the initial design in the optimization of T-junction manifold multiplexers," in Microwave Symposium Digest, 1997., IEEE MTT-S International, 1997, vol. 2, pp. 1025-1028 vol.2.

[3] F. M. Vanin, D. Schmitt, and R. Levy, "Dimensional synthesis for wide-band waveguide filters and diplexers," IEEE Trans. Microw. Theory Tech., vol. 52, no. 11, pp. 2488-2495, Nov. 2004.

[4] P.-S. Kildal, A. Zaman, E. Rajo-Iglesias, E. Alfonso, and A. Valero-Nogueira, "Design and experimental verification of ridge gap waveguide in bed of nails for parallel-plate mode suppression," IET Microw. Antennas Propag., vol. 5, no. 3, pp. 262-270, Feb. 2011.

[5] M. S. Sorkherizi, A. A. Kishk, and M. Saad, "High rejection stacked bandpass filter optimized by group delay response," in Microwave Symposium (IMS), 2015 IEEE MTT-S International, 2015, pp. 1-4.

[6] S. Amari, "Synthesis of cross-coupled resonator filters using an analytical gradient-based optimization technique," IEEE Trans. Microw. Theory Tech., vol. 48, no. 9, pp. 1559-1564, Sep. 2000.

[7] X. Shang, Y. Wang, W. Xia, and M. J. Lancaster, "Novel Multiplexer Topologies Based on All-Resonator Structures," IEEE Trans. Microw. Theory Tech., vol. 61, no. 11, pp. 38383845, Nov. 2013. 